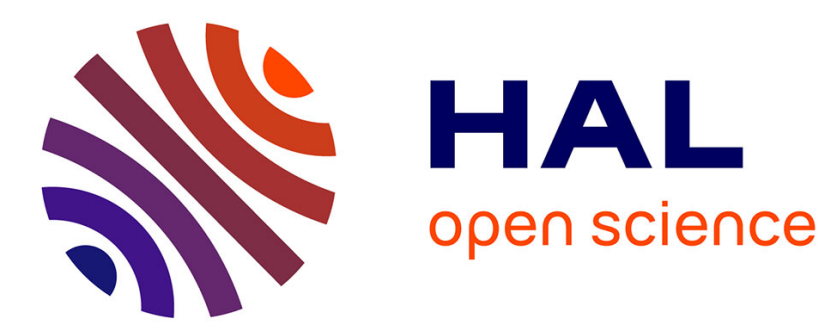

\title{
A PMU-Based Control Scheme for Islanded Operation and Re-synchronization of DER
}

Biswarup Mukherjee, Marcelo de Castro Fernandes, Luigi Vanfretti

\section{To cite this version:}

Biswarup Mukherjee, Marcelo de Castro Fernandes, Luigi Vanfretti. A PMU-Based Control Scheme for Islanded Operation and Re-synchronization of DER. International Journal of Electrical Power \& Energy Systems, 2021, 10.1016/j.ijepes.2021.107217 . hal-03296936

\section{HAL Id: hal-03296936 https://hal.science/hal-03296936}

Submitted on 23 Jul 2021

HAL is a multi-disciplinary open access archive for the deposit and dissemination of scientific research documents, whether they are published or not. The documents may come from teaching and research institutions in France or abroad, or from public or private research centers.
L'archive ouverte pluridisciplinaire HAL, est destinée au dépôt et à la diffusion de documents scientifiques de niveau recherche, publiés ou non, émanant des établissements d'enseignement et de recherche français ou étrangers, des laboratoires publics ou privés. 


\title{
A PMU-Based Control Scheme for Islanded Operation and Re-synchronization of DER
}

\author{
Biswarup Mukherjee ${ }^{\mathrm{a}, *}$, Marcelo de Castro Fernandes ${ }^{\mathrm{b}}$, Luigi Vanfretti ${ }^{\mathrm{b}, *}$ \\ ${ }^{a}$ Centre PERSEE, MINES ParisTech, PSL University, France \\ ${ }^{b}$ ECSE, Rensselaer Polytechnic Institute, Troy, NY, USA
}

\begin{abstract}
This article proposes a novel synchrophasor-based control scheme enabling controlled islanding, islanded operation and automatic re-synchronization of a distributed energy resource (DER) in a distribution network. The performance of the proposed control system is studied using a test power system model. The DER controller uses a centralized architecture, receiving phasor measurement unit (PMU) measurements from both the transmission and distribution grids. In simulation, the frequency control function inside the proposed controller uses frequency estimates calculated using a new formula that exploits the bus voltage in rectangular form (real and imaginary values). The performance of the proposed frequency computation method is studied and compared with the conventional washout filter (WF) approach used by most power system software tools. The study also discusses why unwrapped bus angles are necessary to perform the automatic re-synchronization process. The performance of the proposed controller is evaluated using both deterministic and stochastic load models, allowing the assessment of variability in distribution grids. The implementation of the proposed control scheme and the simulation of the test system is carried out leveraging rich features of Modelica language and the Open-Instance Power System Library (OpenIPSL).
\end{abstract}

Keywords: Power systems modeling, PMU, Controlled islanding, Island operation, Re-synchronization, Power grid, DER, Modelica, OpenIPSL.

\section{Introduction}

\subsection{Motivation}

The concept of operating a smart grid includes the application of smart or intelligent equipment that makes power transmission and distribution secure, 5 reliable and flexible. An important and desired function of smart grids is that of

\footnotetext{
* Corresponding author

Email addresses: biswarup.mukherjee@mines-paristech.fr (Biswarup Mukherjee), decasm3@rpi.edu (Marcelo de Castro Fernandes), vanfrl@rpi.edu (Luigi Vanfretti)
} 
facilitating the autonomous operation of micro-grids [1, 2, 3, where distributed generators (DGs) are connected in the vicinity of, or co-located with, loads. Providing these two functions in today's power system is an important task considering the increasing penetration of DERs.

10 Nowadays, islanding and re-synchronization operations need to be executed occasionally by transmission system operators (TSOs) and/or distribution system operators (DSOs) to maintain proper balance between power generation and consumption. This is often carried out either with proprietary re-synchronizers that lack interoperability, or manually (i.e. without automation) where the ors coordinate changes at the DER site with plant engineers.

This article investigates how to automate the controlled islanding, islanded operation and re-synchronization processes for a synchronous generator-based DER located at the distribution network (DN). To this end, the article presents a centralized control architecture, models the necessary control functions and

20 trip signals that need to be sent to protection relays. The control functions are envisioned to make use of measurements from PMUs [4, 5, 6], located at transmission and distribution networks. This allows the monitoring of synchronization variables across the traditional TSO/DSO operation boundaries and with interoperability assuming they adopt standardized protocols such as IEEE ${ }_{25}$ C37.118.2 [7.

\subsection{Literature review and previous work}

In current power grids, manual and autosynchronizers are widely used to perform re-synchronization in any islanded power network having multiple generators and loads connected to the grid [8. Although manual systems are still used, they are mostly kept as a back-up and automatic systems are preferred due to its inherent greater precision to assess the criteria for synchronization 8, 9, 10. Indeed, in order for synchronization to occur without unwanted consequences, it is necessary to closely monitor synchronization variables: voltage quantities (magnitude, phase-angle) and frequency, and to assure that they are

35 within acceptable limits for the connection of two isolated systems to occur [9, 10.

The problem of re-synchronization is not new and many different creative solutions have been proposed in the literature recently, since the topic has gained attention due to the bolstering deployment of DERs. In fact, a virtual syn40 chronous machine (VSM)-based control strategy is presented in [1], allowing microgrids to operate in both grid-connected and islanded without control loop reconfiguration. The proposed strategy acts on reducing frequency error from a VSM reference and phase-angle difference for a seamless re-connection of islanded grids. In [12, a novel strategy for the smooth transition of DERs from 45 grid-connected to islanded operation modes is investigated. In that paper, the proposed control action is independent on the islanding detection time. Moreover, distributed control of islanded microgrids are presented and investigated in 13 and in [14] for increased resiliency. The distributed approach in [13] allows microgrids to perform primary, secondary and tertiary control actions 50 locally, reducing its dependency on external measurement. On the other hand, 
[14] uses the distributed architecture but proposes adaptive protocols that can compensate any adverse effect that might occur to the sensor.

It is important to observe that the previous solutions only contemplated the usage of local measurements. This is mainly because most of the previous work 55 use inverter-based energy resources (IBRs) which are able to derive local frequency measurements from their phased-locked loops (PLLs), so that controlled islanding and post reconnection procedures can be performed quickly and precisely [15, 16. However, there are different studies in the literature that present how remote sensing can be used for islanding and reconnection procedures, and

60 this becomes attractive for more conventional generators that would also need to operate under such conditions. These remote sensing quantities may come from PMUs and, studies on the application for synchrophasors for these purposes has been proposed in [17, 18, 19. In [20, a study analyzes how synchrophasors could be used to create a controlled islanding in the Uruguayan power system 65 as a protective measure. As an example of a microgrid-related work, a strategy leveraging the remote sensing of both voltage and frequency quantities is proposed in 21]. The control actions for automatic re-connection after an intentional islanding of a microgrid are based on conventional synchronism check relays and they are demonstrated to result in a smooth procedure.

The usage of both local signals and synchrophasor measurements would, then, be essential for a centralized architecture to function properly. In fact, it is absolutely necessary to adequately model the measurements, especially the frequency. A common approach in power systems tools is to use a WF in order to model the estimate of the bus frequency 22. However, this well-known 75 solution is prone to numerical issues [22] and, therefore, it is necessary to find an alternative approach. In 23 the frequency divider approach is presented and it is compared to the WF approach in 22, while this method is attractive, it is not well suited for generic modeling tools and has only been implemented in the tool reported in [22]. In this paper, a more practical and simpler approach s is proposed based on rectangular components of the voltage phasor. This is attractive because it becomes straightforward to implement in general purpose tools, but also, it follows the same principle that actual PMUs use to estimate the frequency internally, i.e. they estimate frequency based on the voltage angle [7.

85 Furthermore, in previous work 24, the authors proposed a control system architecture for islanded-operation, based on PMU measurements; however, that architecture did not provide the means to perform automatic resynchronization functions and to provide trip signals to relays to perform the required re-closing of the circuit breaker. In addition, the authors modeled an automatic re-synchronization function for DER generators [25], but it did not include an islanded-operation control function that would allow the islanded system to self-regulate its frequency. Therefore, this work proposes an integrated control architecture for both controlled islanding, islanded operation and automatic re-synchronization. The proposed controller, if adopted in the field,

95 can operate taking measurements from synchrophasor data, hence providing an interoperable solution as compared to today's common practices. 


\subsection{Contributions}

The main contributions of this article are:

(i) For modeling and simulation purposes, a new frequency estimate computation method based on the bus voltage phasor data in rectangular form is proposed. This aims to emulate frequency measurements, such as that obtained from PMUs, when used in a closed loop controller. For comparison, the Modelica 26] implementation of the conventional WF model [22, 23] for frequency computation used in most power system tools, is presented. The computed frequency values obtained using both the techniques are analyzed and compared.

(ii) An integrated control system architecture is proposed, which is interfaced in cascade with a turbine's governor and excitation controls (i.e. voltage regulator), without replacing the existing generator control structure. Within this control architecture, a control scheme for controlled islanding, islanded operation and autonomous re-synchronization is proposed.

(iii) Taking advantage of PMU measurements, an angle difference control function is proposed to influence the phase angle difference during the grid re-connection process. It is modelled inside the proposed control architecture, and compliments traditional voltage and frequency control functions.

(iv) The use of advanced object-oriented equation-based modeling language features from Modelica are exploited and illustrated, giving an example of how such advanced language features can facilitate design and analysis of new control functionalities, i.e. controlled islanding, islanded operation and re-synchronization.

(v) The performance of the control system is evaluated considering both deterministic, and stochastic load models. This analysis allows to understand the feasibility of the proposed control system's operation under different levels of time-domain variability.

(vi) Finally, to extend the analysis for effectiveness and robustness of the proposed control scheme, aspects related to the use of the PMUs for feedback are considered. The performance of the proposed scheme is evaluated for different values of the PMU reporting rate and PMU data transmission delay.

The reminder of this paper is organized as follows: In Section II, for simulation purposes, a new frequency computation approach is proposed and described along with the conventional method used by power system simulators. Section III presents the proposed control architecture and its Modelica implementation.

135 The power system model and simulation set-up is presented in Section IV. Four case studies are presented in Section V, while the conclusions summarizing the paper results are presented in Section VI. 


\section{Frequency Computation}

When the distribution grid is disconnected from the bulk transmission grid 140 to become an islanded system, the phase angle of the bus voltage in the distribution grid starts deviating from the main transmission system reference. As a result, the distribution network frequency also deviates from the transmission grid frequency. The frequency at the distribution grid can be controlled by a DER, if equipped with a controller for this purpose. If the frequency at the distribution grid is monitored with a PMU, this measurement can be used to control the distribution network's frequency. However, PMUs do not directly measure frequency. Instead, frequency is a derived quantity, obtained from estimates that use the bus voltage's rate of change in their phase angles [27. The frequency $f(t)$ estimation can be expressed as:

$$
f(t)=\frac{1}{2 \pi} \times \frac{d \theta(t)}{d t}=f_{0}+\frac{1}{2 \pi} \times \frac{d \phi(t)}{d t},
$$

where the phase function $\theta(t)=2 \pi f_{0} t+\phi(t)$ includes both frequency and phase angle.

\subsection{Theory and implementation}

In this work, it is assumed that PMUs estimate frequency from the bus voltage angles. The PMUs are assumed to be located at both the transmission and distribution network will provide frequency signals to the proposed control system. However, this has to be carefully considered in modeling and simulation 22, 23, because if the bus frequency is calculated from the bus angle directly, angle wrapping will produce sharp discontinuities in the derived frequency values. To overcome this issue and to provide useful and adequate frequency signals for control purposes, a new method is proposed herein. Here we have considered only two PMUs, one at the transmission grid and the other one at the distribution grid where the DER is located. Note that for the islanded operation, only one PMU is necessary in theory, but the re-synchronization requires both the PMUs. Consequently, we assume no other PMUs are installed 165 throughout the system. In practice, at a minimum, at least two PMUs at the transmission grid and two PMUs at the distribution grid, all at different locations, should be utilized.

Let $V_{i}$ and $V_{r}$ be the imaginary and real parts of complex bus voltage $V$, then we have that

$$
V=\sqrt{V_{i}^{2}+V_{r}^{2}}
$$

170 and the bus angle $\theta$ can be expressed as in (3).

$$
\theta=\arctan \frac{V_{i}}{V_{r}}
$$


Therefore the angular frequency $\omega=2 \pi f$ can be estimated taking the first order derivative of the bus angle $\theta$, as presented in (4).

$$
\dot{\theta}=\omega=\frac{V_{r} \dot{V}_{i}-V_{i} \dot{V}_{r}}{V_{i}^{2}+V_{r}^{2}}
$$

Note that for the quantity $\omega$ to be in per unit, it is necessary to divide it by the base frequency (i.e. $2 \pi f_{0}$ ). Equation (4) was implemented using Modelica language within a frequency computation model, as follows:

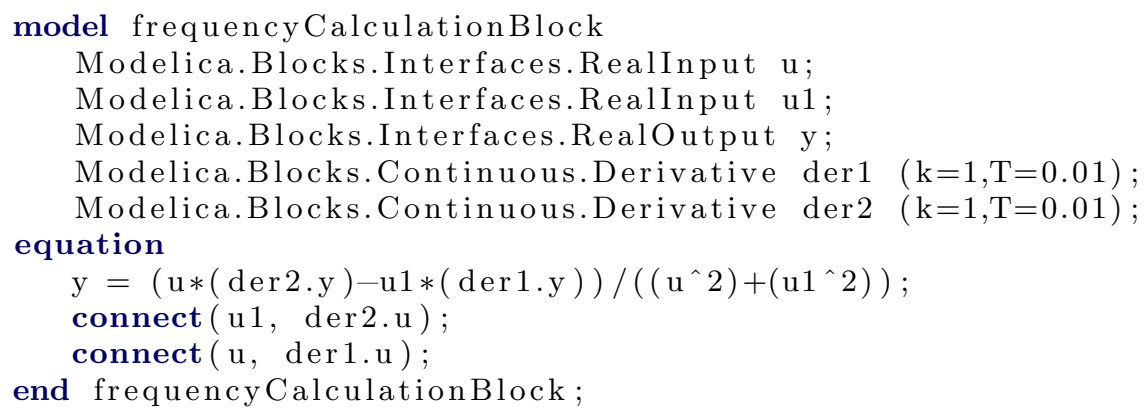

Note that the derivative function blocks, denoted by der1 and der2, use a gain value of $\mathrm{k}=1$ and a time constant of $\mathrm{T}=0.01 \mathrm{sec}$. Since the proposed computation method relies on the real and imaginary values of complex bus voltage, the method is independent of rate of change of voltage phase angle. This makes the proposed method advantageous over the WF model, presented in the next section.

\subsection{Washout Filter implementation in Modelica}

In traditional power systems tools [28, the WF model is used to estimate the grid frequency from the bus voltage phase angle. This model computes bus frequency by passing the voltage phase angle through a derivative block and a first order filter. This paper presents the Modelica implementation of the WF model shown in Fig. 1, which is deployed in the power system model.

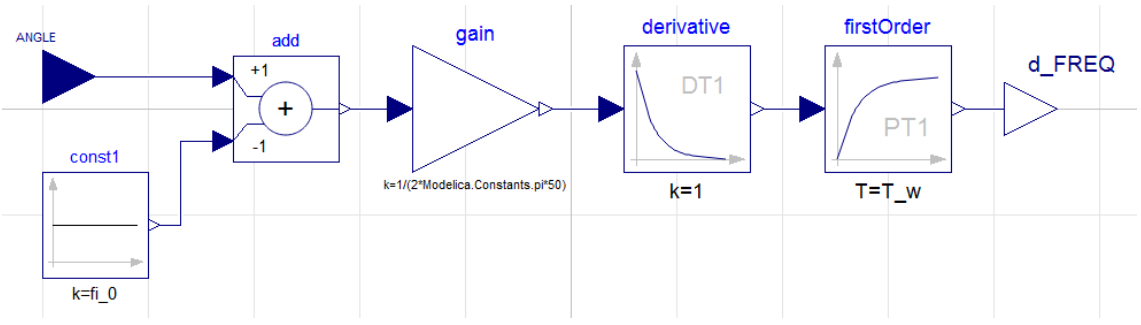

Figure 1: Washout Filter (WF) implementation in Modelica.

The parameter used for the WF model are as follows: $k=1, T_{-} f=1$ sec and $T_{-} w$ ${ }_{200}=2$ sec, where $T_{\_} f$ and $T_{\_} w$ are the time constants of the derivative and first 
order filter blocks, respectively. The gain $\mathrm{k}$ remains same for both the derivative block and the first order filter models.

In this work we have performed the simulations using phasor time-domain modeling framework, it is to exploit both the components (real and imaginary) of the complex bus voltage phasor to compute the frequency, similar to the way this is performed by PMUs which derive the frequency from the bus voltage angle. Note that this approach is much different than those used in power electronic systems which derive the frequency from a PLL. Hence, because of the modeling framework and to be consistent with how PMUs derive frequencies, 210 we propose the frequency computation using bus voltage phasor components. Hence, in Section 2.1 we propose an alternative and more practical approach over the WF model.

\section{Proposed Controller Architecture and Functions}

\subsection{Control architecture}

The proposed control system architecture and internal functions for control islanding, islanded operation and automatic re-synchronization is presented in Fig. 2. The overall structure including sensing (PMUs), data communications and the controller's analog and digital outputs is shown in Fig. 2a, while the internal functions are presented in Fig. 2b. The controller has two main modes of 220 operation: (1) controlled islanding and islanded operation (highlighted by green dotted line), (2) automatic re-synchronization (highlighted by orange dotted line).

The mode for islanded operation requires a PI controller, that can be used to restore the frequency deviation from the nominal value after the system is separated from the main grid. This function can be activated as soon as the island is detected.

The control mode for automatic re-synchronization consists of three major functions or units: computation unit, activation unit and control unit. The synchronizing variables are determined from two PMUs each at different remote 230 sub-stations, one located at the transmission network and one in the distribution network. The synchronization variables are: voltage difference $(\Delta \mathrm{V})$, frequency difference $(\Delta \mathrm{f})$ and angle difference $(\Delta \theta)$. These three synchronization variables are determined by the computation unit within the centralized controller at the DER. The activation unit plays a key role in activating three different

235 controllers (voltage controller, frequency controller and an angle-difference controller) following a sequential decision making process, that checks thresholds for three different synchronization variables $(\Delta \mathrm{V}, \Delta \mathrm{f}, \Delta \theta)$. When all three synchronization variables are below their thresholds after a given amount of time, the controller will send a trip signal to a relay to close the corresponding circuit breaker and to complete the re-synchronization process.

\subsection{Modeling of the automated re-synchronization control mode}

Figure 3 summarizes the algorithm for the sequential control logic for resynchronization. During the re-synchronization process, the voltage and fre- 


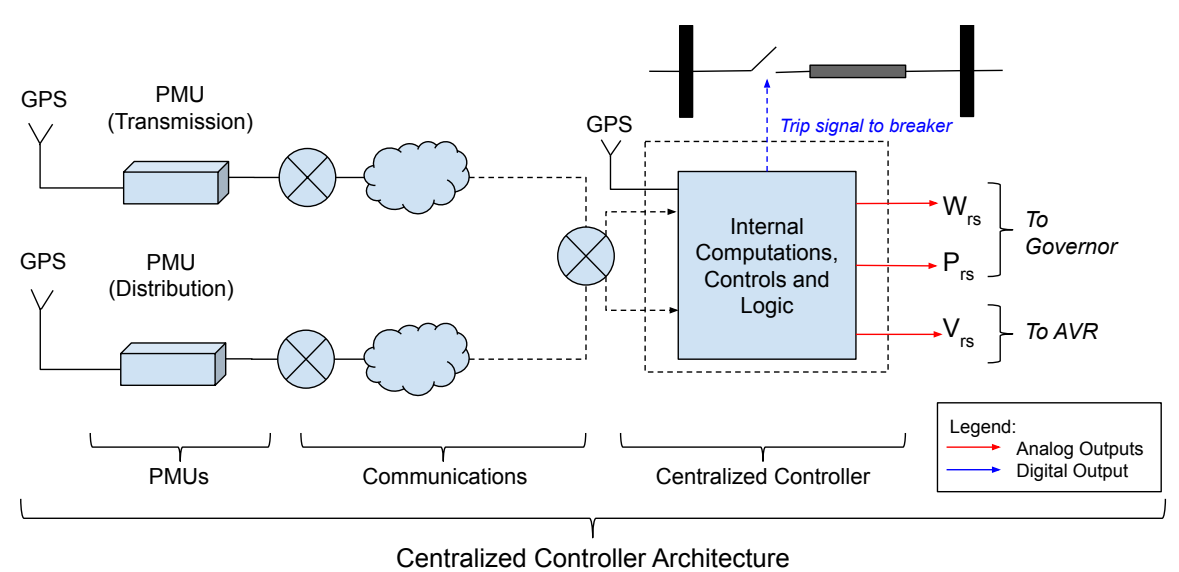

(a) Centralized controller architecture at the DER using remote PMU measurements.

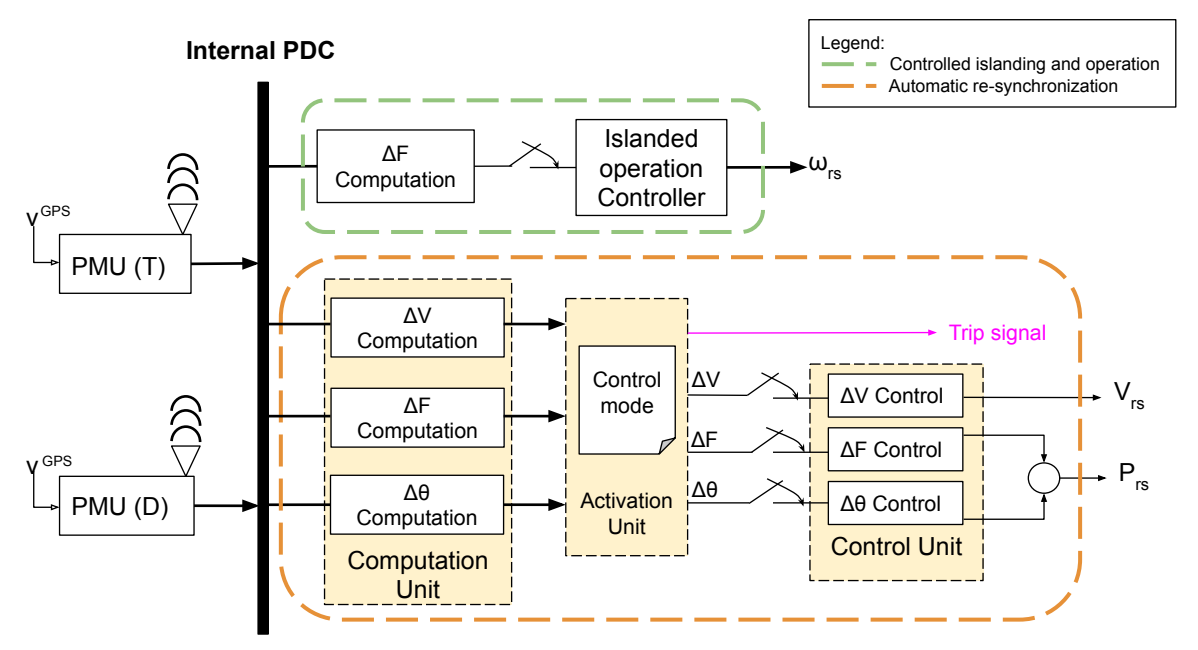

(b) Internal control functions.

Figure 2: Controller Architecture and Functions.

quency control functions can be modeled using PI controllers, as reported in

21]. To control $\Delta \theta$, which is the third re-synchronization variable, an angle difference controller that requires a PID block is proposed herein. In the sequel, each individual control function is summarized, followed by a discussion on how they are used in the sequential control logic. Implementation aspects exploiting the Modelica language are summarized also.

\subsubsection{Modeling}

The voltage controller output is defined by Eq. (5), where $\mathrm{x}$ represents the Boolean input signal, (start_voltage) that activates the controller when the re-synchronization process initiates. 


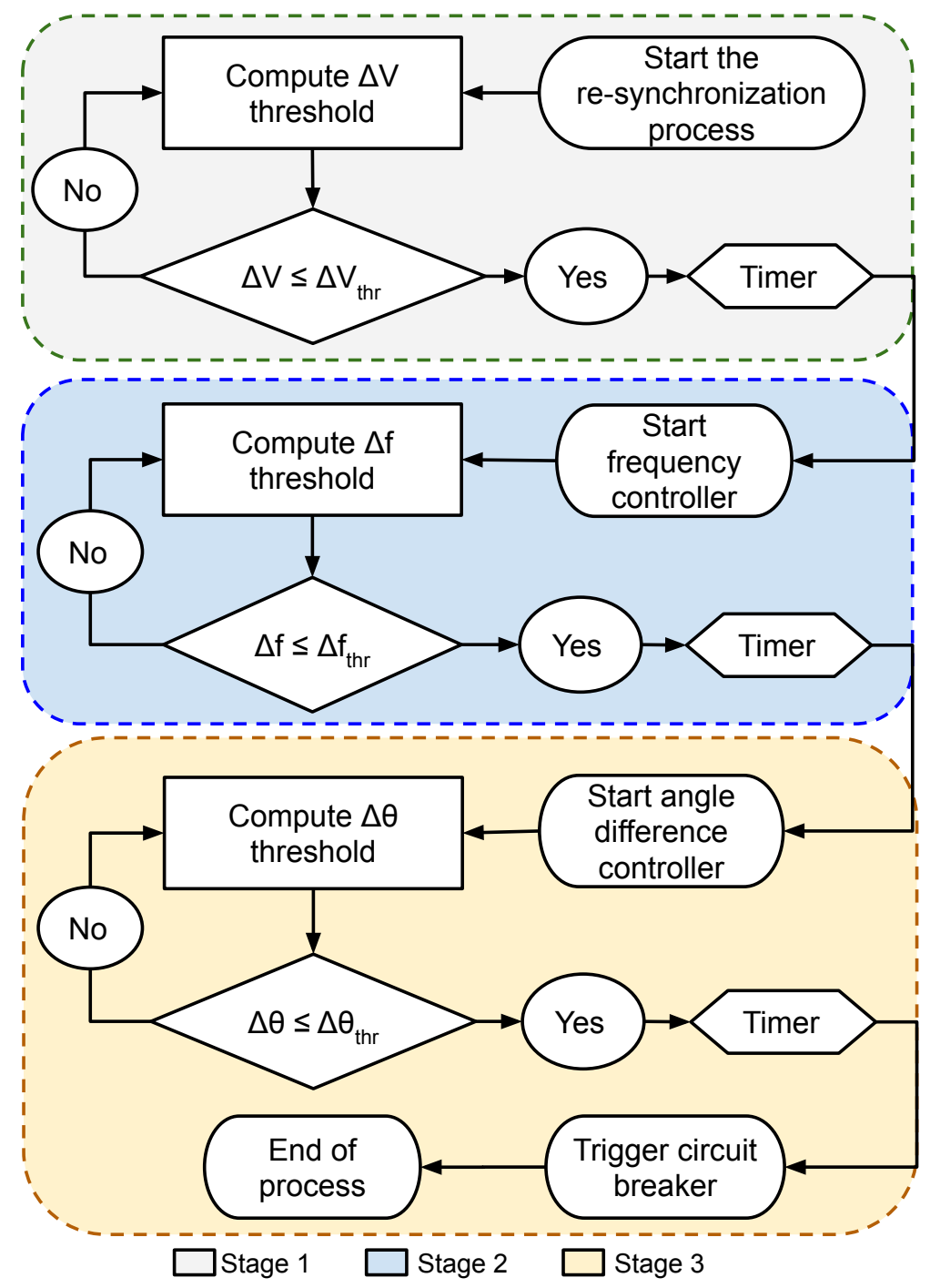

Figure 3: Sequential control logic for re-synchronization.

$$
y(\mathbf{x})= \begin{cases}\left(K_{P_{V}} \Delta \mathrm{V}+K_{I_{V}} \int_{0}^{t} \Delta \mathrm{V} d t\right), & \text { if } \mathrm{x}=\text { true } \\ 0, & \text { otherwise }\end{cases}
$$

where $K_{P_{V}}$ and $K_{I_{V}}$ are the proportional and derivative gains of the voltage 255 control function. The output of the voltage controller is applied to the automatic voltage regulator (AVR) error signal, which controls the generator field voltage. If the Boolean input signal, start_voltage, is true, the computed volt- 
age difference is fed to the PI block. If this Boolean signal is false, the output of the controller becomes zero.

The output of the frequency controller modulates the turbine's power error signal. Similar to voltage controller, the frequency controller output can be defined by Equation (6):

$$
y(\mathbf{x})= \begin{cases}\left(K_{P_{f}} \Delta \mathrm{f}+K_{I_{f}} \int_{0}^{t} \Delta \mathrm{f} d t\right), & \text { if } \mathrm{x}=\text { true } \\ 0, & \text { otherwise }\end{cases}
$$

where $K_{P_{f}}$ and $K_{I_{f}}$ represent the proportional and integral gains of the frequency control functions, respectively. The Boolean input signal $\mathrm{x}$ activates the frequency controller after the voltage bounds are checked successfully. When all three $(\Delta \mathrm{V}, \Delta \mathrm{f}$ and $\Delta \theta)$ bounds are checked successfully, the frequency controller is deactivated.

To monitor and control the phase angle of the bus voltage an angle-difference controller is used. The angle controller uses a PID function whose input is the 270 unwrapped angle difference estimated from the transmission and distribution side bus voltage angles. The output of this controller corresponds to the following equation:

$$
y(\mathbf{x})= \begin{cases}\left(K_{P_{\theta}} \Delta \theta+K_{I_{\theta}} \int_{0}^{t} \Delta \theta d t+K_{D_{\theta}} \frac{d \Delta \theta}{d t}\right), & \text { if } \mathrm{x}=\text { true } \\ 0, & \text { otherwise }\end{cases}
$$

where $K_{P_{\theta}}, K_{I_{\theta}}$ and $K_{D_{\theta}}$ represent the proportional, integral and derivative gain of the angle-difference control function respectively.

When the re-synchronization process begins, a signal activates the control logic inside the activation unit. The outputs of the re-synchronization controller can be expressed using the following equations:

$$
\begin{aligned}
V_{r_{s}} & =\left(K_{P_{V}} \Delta V+K_{I_{V}} \int_{0}^{t} \Delta V d t\right) \\
P_{r_{s}} & =f_{P}+f_{\theta} \\
f_{P} & =\left(K_{P_{f}} \Delta f+K_{I_{f}} \int_{0}^{t} \Delta f d t\right) \\
f_{\theta} & =\left(K_{P_{\theta}} \Delta \theta+K_{I_{\theta}} \int_{0}^{t} \Delta \theta d t+K_{D_{\theta}} \frac{d \Delta \theta}{d t}\right)
\end{aligned}
$$

where $V_{r_{s}}$ is applied to the supplementary input signal of the AVR, $P_{r_{s}}$ is applied to the turbine's power set-point, while $f_{P}$ and $f_{\theta}$ are applied to the governor's speed set-point. The controllers will change these outputs with the goal of minimizing the synchronization variables.

When the synchronization variables $(\Delta \mathrm{V}, \Delta \mathrm{f}$ and $\Delta \theta)$ are below their thresh280 olds for a pre-defined amount of time, the Boolean output signal is sent to a protection relay that would close the circuit breakers of the tie-line, which 
finishes the re-synchronization of the islanded distribution network with the transmission network.

The sequential control logic in Fig. 3 was implemented within activation unit, where limit checking blocks are modeled to monitor the synchronizing variables, with the following thresholds:

$$
\begin{aligned}
& \Delta V_{\text {min }} \leq \Delta V \leq \Delta V_{\text {max }} \\
& \Delta f_{\text {min }} \leq \Delta f \leq \Delta f_{\text {max }} \\
& \Delta \theta_{\text {min }} \leq \Delta \theta \leq \Delta \theta_{\text {max }}
\end{aligned}
$$

where the $\Delta x_{\min }$ and $\Delta x_{\max }$ values for $x: \mathrm{V}, \mathrm{f} \& \theta$ need to be determined equivalent impedance between the tie-line linking the transmission and distribution network. This implies that ideally, the re-connection should be performed when there is minimal power being fed through that line, resulting in a smooth coupling between the systems. Similarly, $\Delta V_{\max }$ would be the largest possible voltage drop through that li
error, for example $\pm 0.01 \mathrm{~Hz}$

\subsubsection{Modelica implementation}

To avoid the numerical issues during simulation, advanced Modelica language features are used to handle phase angle wrapping before it was applied to the $\Delta \theta$ controller. The controller uses the updated difference value theta_diff_new estimated from the angular difference between the transmission and distribution network bus voltages. The calculation of theta_diff_new uses different Modelica operators such as homotopy, smooth, noEvent [29, 30, as follows: end ;

equation

theta_diff $=(-$ B6.angle $)+B 4$.angle;

theta_diff_new $=$ homotopy $($ actual $=\operatorname{smooth}(0, \operatorname{noEvent}($ if

theta_diff $>180$ then theta_diff -360 else if

theta-diff $<(-180)$ then theta_diff +360 else

theta_diff)), simplified $=$ theta_diff $)$;

connect(theta_diff_new, G22.theta_diff);

For the homotopy operator the argument simplified = theta_diff implies that the initialization process uses this simplified expression, thereby making the initialization process more robust. Meanwhile, the smooth operator is provided an argument 0 to indicate that the variable theta_diff_new is discontinuous, i.e. non-differentiable. This allows to model the actual behavior for this control input variable as seen in the field [31, where the measured phase angles will be discontinous when the system undergoes a line trip and similar discrete changes.

315 Finally, the noEvent operator helps with event suppression. This minimizes the number of states events that can arise during simulation. State events require reinitialization of the differential-and-algebraic equation model, and thus, degrade simulation performance 32. Therefore, noEvent helps avoiding such events, improving simulation performance. 


\subsection{Modeling of the controlled islanding and island operation mode}

Once the distribution network is islanded, both power balance and frequency would need to be controlled by at least one of the DERs in the network. In this work, only the case when a single DER is located in the islanded portion of the network is considered. It is assumed that the DER consists of a conventional synchronous generator with both speed and voltage controllers. To achieve autonomous operation as an island, the DER is equipped with the proposed controller architecture described above. To achieve autonomous island operation, the proposed controller at the DER would be equipped with an ICL (Intelligent Control Logic) for islanded operation, which that can use real-time frequency measurements from PMUs that can be placed in any location within the distribution grid where the DER is present.

The ICL is modeled within the distribution network generator (G22) (see Figs. 5 and 60 that uses a PI controller in order to maintain zero frequency deviation when the islanding occurs. The output of the PI controller within ICL can be expressed by Equation (10), where $K_{P_{I}}$ and $K_{I_{I}}$ are the proportional and integral gain respectively of this PI function and $\Delta \Omega_{L}$ represents the error in the distribution network frequency from its nominal value. The implemented algorithm within ICL disables the PI controller modeled inside when the automatic re-synchronization process begins and, at the same time, the ResynchUnit initiates the voltage control action. Hence, the re-synchronization maneuvering process for an isolated distribution grid is carried out autonomously.

$$
\Omega_{r_{s}}= \begin{cases}\left(K_{P_{I}} \Delta \Omega_{L}+K_{I_{I}} \int_{0}^{t} \Delta \Omega_{L} d t\right), & \text { if } \mathrm{x}=\text { true } \\ 0, & \text { otherwise }\end{cases}
$$

\subsection{Modeling of the control mode transitions}

The transitions between different control modes used by the proposed controller are shown in Fig. 4. Note that it shows labels indicating how the scheme executes the transitions from one control mode to another.

For illustration, assume that a DER with the proposed control system is islanded. The control system will either detect an island or the island will be created and activate the islanded operation controller (see label (A) in Fig. 4). After the system has been operated in islanded mode under acceptable conditions, the re-synchronization controller can be activated as shown by (B) in Fig. 4. which concurrently disables the islanded operation controller (see (C) of Fig. 4). As soon as the re-synchronization controller starts operating, the sequential control mode logic (see Fig. 3) is activated. Naturally, once the re-synchronization process is completed, the re-synchronization controller is deactivated, leaving only the conventional controls (AVR and Governor) to drive the DER. 


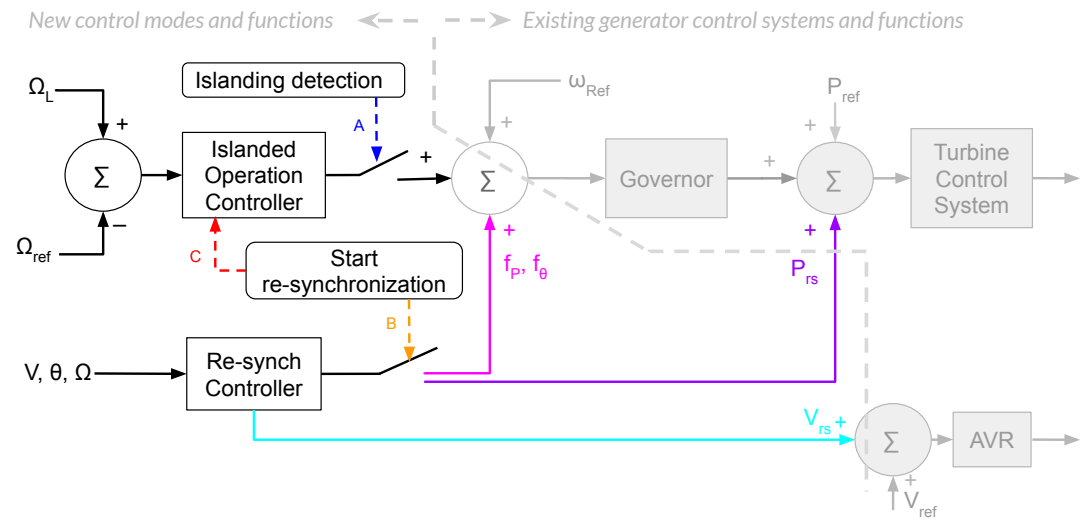

Figure 4: Control scheme transitions between modes of operation. Grey lines and fill colors indicate existing conventional generator control system functions, while new functions and signals are shown in black and other colors.

\section{Power System Modeling and Simulation Set-up Implementation}

The power system model, shown in Fig. 5, was implemented using Modelica language and the OpenIPSL [33, 34] in Dymola [32, 35] in order to study the performance of the proposed automatic re-synchronization controller. The controller architecture was placed inside the DER generator model (G22) at the distribution network, as shown in Fig. 6. The transmission network generator model (G1) uses a GENSAL block that corresponds to the synchronous generator model with salient poles and a simple excitation control system represented by the SEXS model. To start the islanding process, the entire system frequency was perturbed by introducing a speed change in the hydro-turbine and governor system model (HYGOV) inside G1. The HYGOV is a standard hydro turbine governor model, and it is the same as the one in PSS/E.

For the DER at the distribution network the different components of the plant are shown in Fig. 6. An IEEESGO block was used to model the gas turbine and governor system within G22. The IEESGO model is an IEEE standard model for turbine-governor systems, which can be used to represent both reheat steam turbines or simplified gas turbines. To model the excitation control system the SEXS block was used. The other two components of G22 are the ResynchUnit and the ICL blocks, as explained in Section III.

When the islanding begins, the controlled circuit breaker (CB2) islands the distribution grid from the transmission network and at the same time a signal is sent to the ICL, activating a PI block within this model. The algorithm to automate the switching from islanded mode to re-synch mode and the operation 


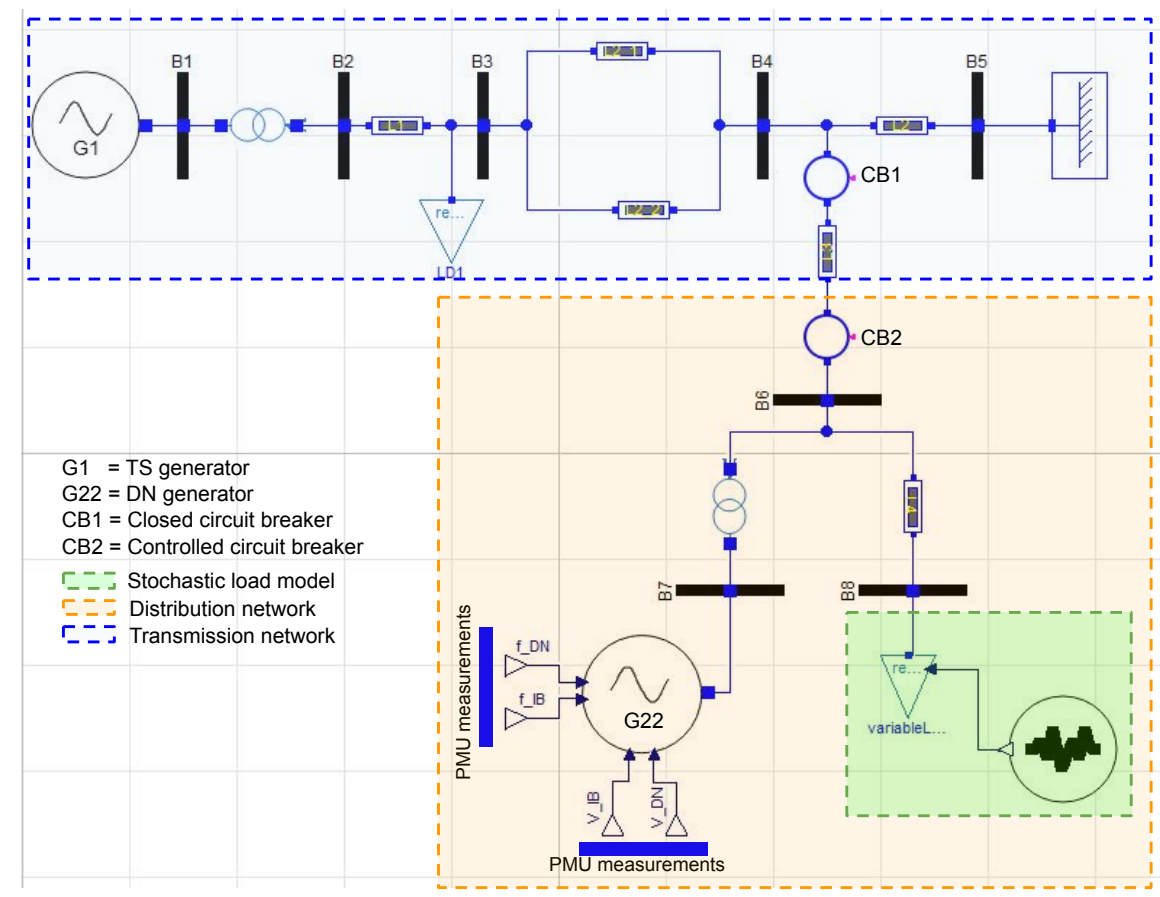

Figure 5: The power system model.

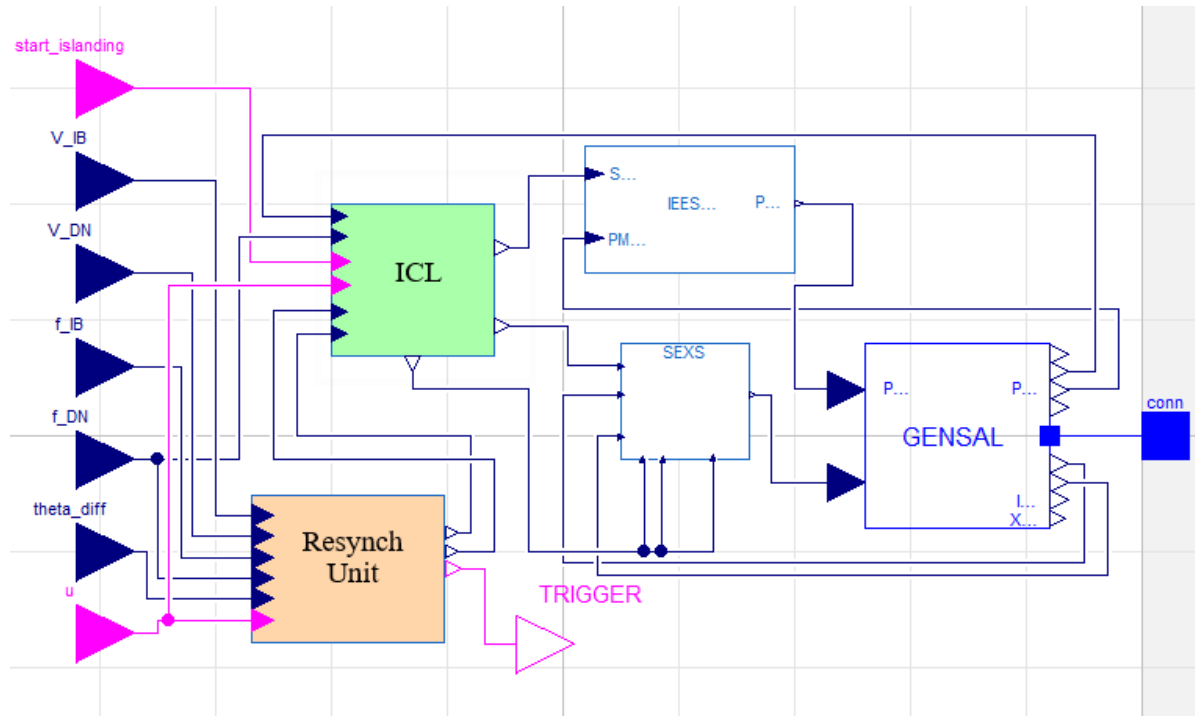

Figure 6: Centralized control structure implementation within the generator model (G22). 


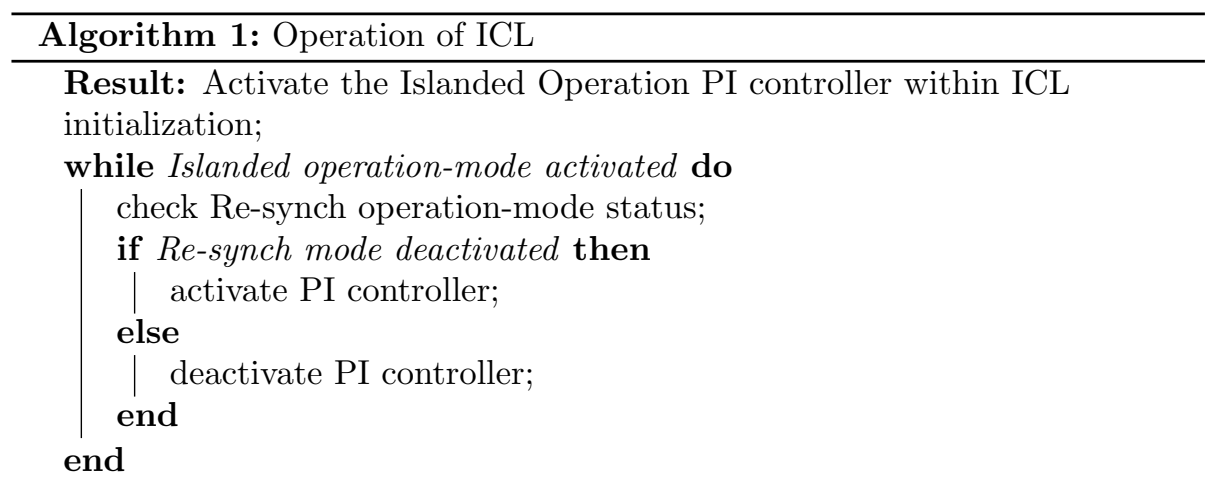

Modeling of the stochastic variability of the aggregate load was carried out by injection of white noise to the PQ load model, as highlighted in green in Fig. 5. Note that white noise generates a signal having normal distribution uses the real and imaginary parts of the same bus (B6) voltage to compute the distribution network frequency.

${ }^{1}$ For brevity the detailed modeling of this block is not presented here. 


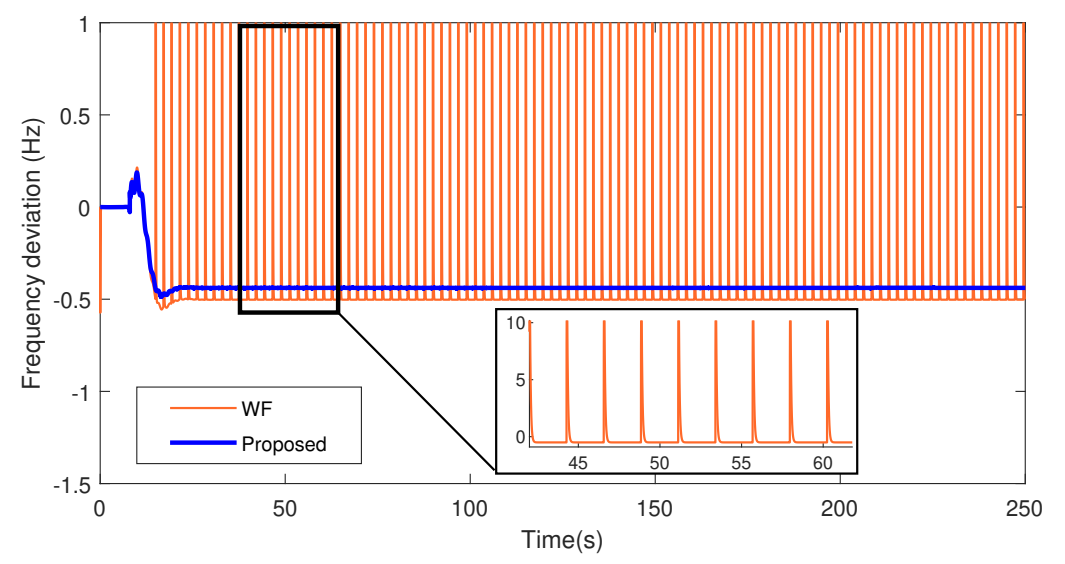

Figure 7: Frequency computation comparison between the proposed and the WF approaches.

Figure 7 shows that the WF model introduces transients in the frequency calculation (red trace) due to bus angle switching, whereas the proposed technique provides a smooth frequency output (blue trace).

For both the models the outputs of the frequency controller are plotted in Fig. 8. The plot shows, due to frequency calculation provided by WF model, the frequency controller fails to operate successfully and introduces spikes or 415 transients (red trace) in its output. On the other hand, the blue trace shows that the controller operates successfully by producing the expected steady state error signal, when it has the input frequency computation using the proposed approach. This study shows the advantage of using the proposed frequency computation technique over the conventional WF method for frequency computation.

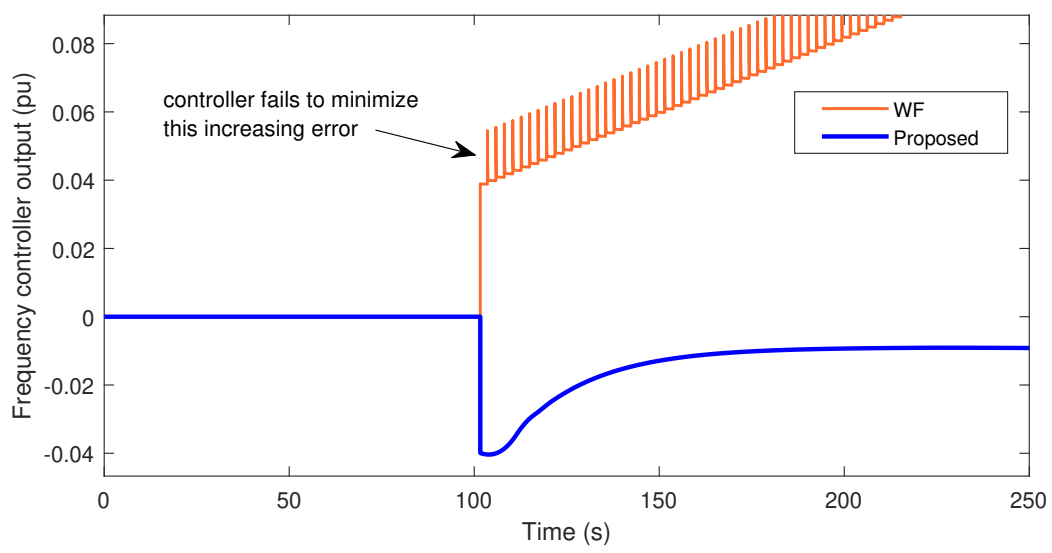

Figure 8: Output of frequency controller with both proposed and WF method. 


\subsection{Case 2: Controlled scheme performance analysis}

This case study was performed to evaluate the performance of the proposed control scheme for both PQ load and an infinite bus connected to the transmission network side of the developed power system model, discussed in the previous section. Simulation results are plotted in Fig. 9 16 for 5 MW power dispatch from the distribution network generator. Figure 9 plots the distri-

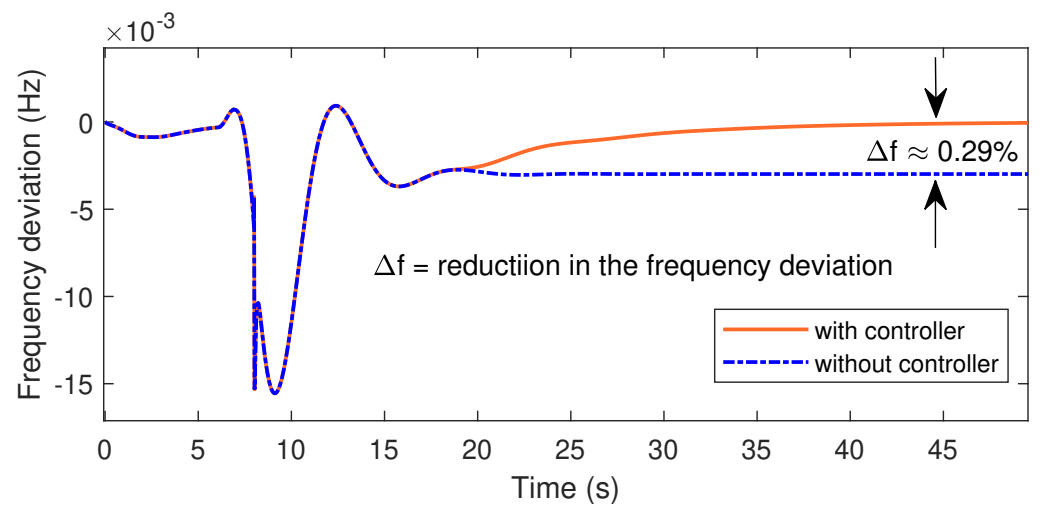

Figure 9: Controlled islanding vs. uncontrolled islanding.

bution network frequency deviation for both a controlled and an uncontrolled islanding event executed before the automatic re-synchronization process by ResynchUnit. Observe from Fig. 9 that the distribution network frequency deviation returns to zero (the red trace) for the controlled islanding operation, while in case of uncontrolled islanding the control architecture fails to do so (shown by the blue dotted trace). This demonstrates the functionality of the islanded operation controller as shown in Fig. 4 .

Figure 10 depicts the frequency deviation in both transmission and distri435 bution network when a PQ load is connected to transmission grid at bus B5. This is performed to illustrate the difference of modeling the reminder of the network with an infinite bus and how that influences the system behavior. Figure 10 clearly exhibits how frequency controller acts to minimize the frequency difference when the re-synchronization process initiates. Note that when the re-synch starts, the control functions are applied to first minimize the voltage difference $(\Delta V)$ (see Fig. 11), followed by the $\Delta \mathrm{f}$ control. Note that because the transmission side (TS) does not have automatic generation control (AGC), the frequency deviates according to G1's droop, and the $\Delta \mathrm{f}$ controller drives the G22 to match the distribution network frequency with the TS frequency 445 instead of returning it to a fixed reference frequency. This is important as the TS frequency is never exactly at its reference value (i.e. 50 or $60 \mathrm{~Hz}$ ), which means that the proposed controller will be able to track the TS instead of a fixed frequency reference.

Figures 12 and 13 show the frequency deviation and voltage profile of the power system due to the re-synchronization operation when the transmission 


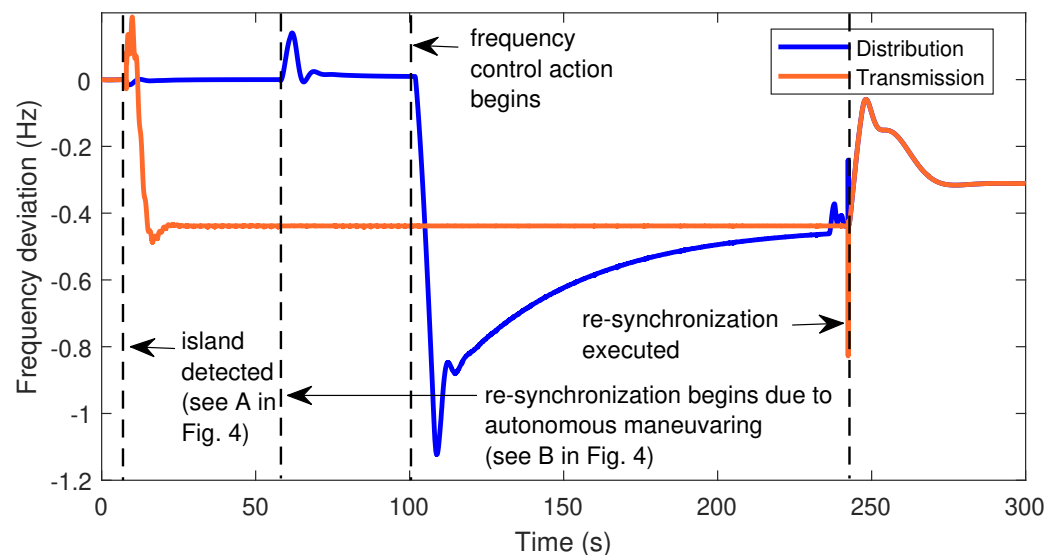

Figure 10: Frequency deviation in both transmission and distribution network for a PQ load model connected to the transmission network.

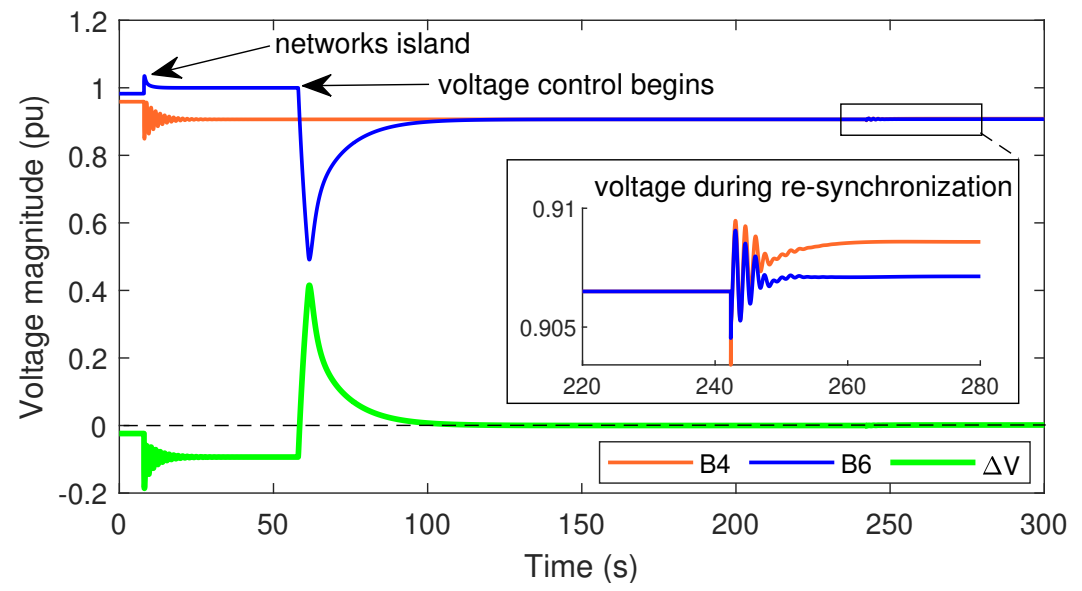

Figure 11: Transmission and distribution network bus voltages for a PQ load model connected to the transmission network.

grid remains connected to an infinite bus. Observe from Fig. 12 that as the transmission network frequency swings, but returns to $50 \mathrm{~Hz}$ after both the islanding and the re-synchronization. Hence, this is an ideal scenario; because the transmission grid includes an infinite bus that establishes the reference fre455 quency in the TS, and, as a result, the distribution grid frequency returns at $50 / 60 \mathrm{~Hz}$ before and after the re-synchronization process.

From Fig. 13 it can be observed that as soon as the islanding begins, the bus voltage levels at B4 and B6 are affected, as a result there exist the voltage difference until the voltage control action begins. After voltage control action 
ends, the $\Delta V \approx 0$ as it can be observed from the green trace. This ensures that the voltage controller reduces the control error to the desired threshold. It can also be observed from the simulation result that the re-synchronization process does not affect the bus voltages or introduce switching transients, this is due to the stiffness of the infinite bus at the transmission network.

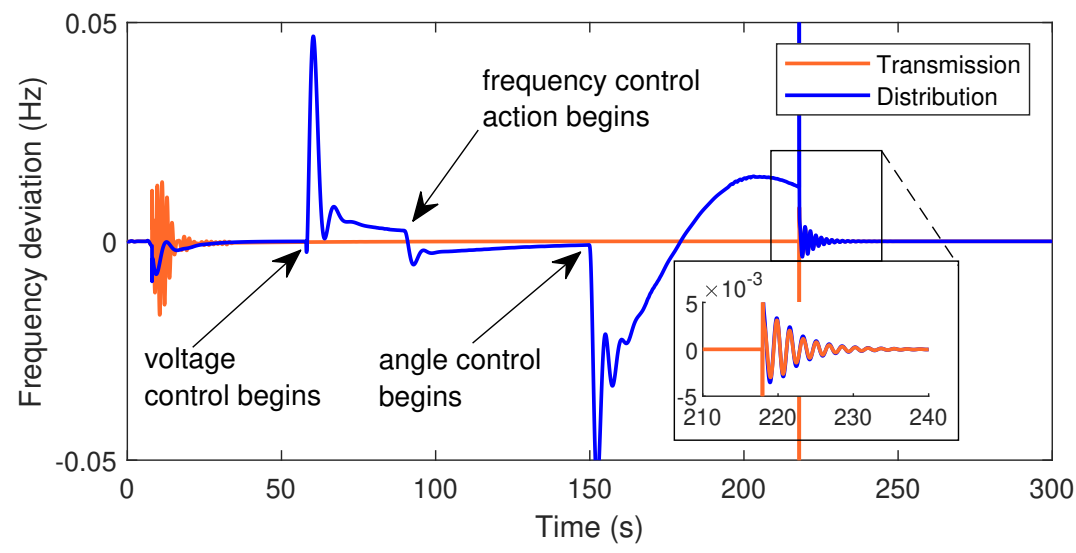

Figure 12: Frequency deviation in both transmission and distribution network for an infinite bus connected to transmission grid.

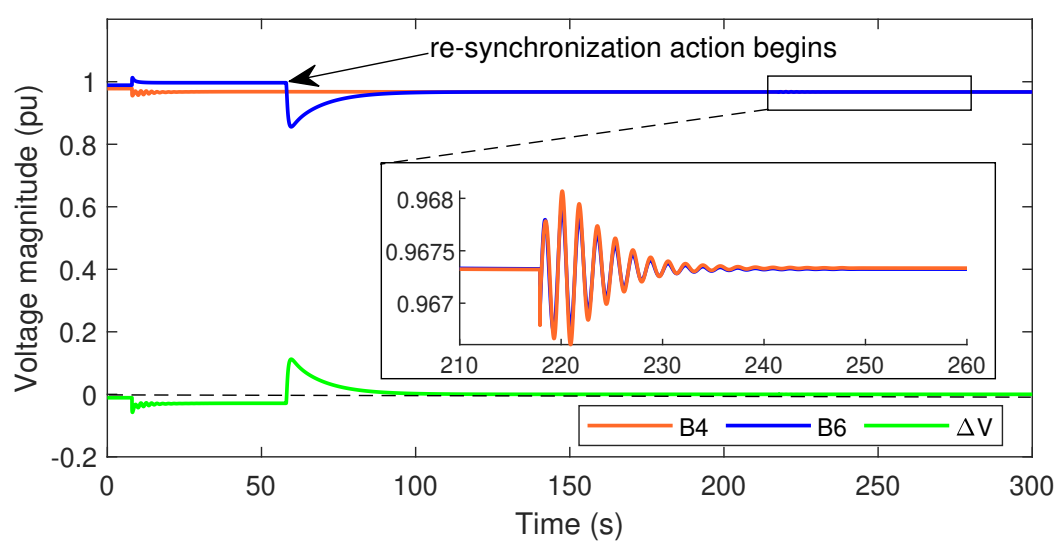

Figure 13: Transmission and distribution network bus voltages for an infinite bus connected to transmission grid.

Figures $14-16$ are plotted to compare the performance of the proposed control scheme for both IEESGO and HYGOV models. The results validate the efficacy of the proposed scheme when two different turbine and governor systems are modeled within the distribution generator. Figure 14 shows the frequency deviation in the distribution grid for different gains (i.e. $K_{P_{V}}$ and $K_{I_{V}}$ ) of the 

IEESGO the transient performance can be improved (as seen from the red trace, the overshoot is reduced $\approx 16 \%$ ) by tuning the control parameters. A similar performance can be noticed in Fig. 15 when the transmission network is connected to an infinite bus. Here we observe that the change in gain parameters

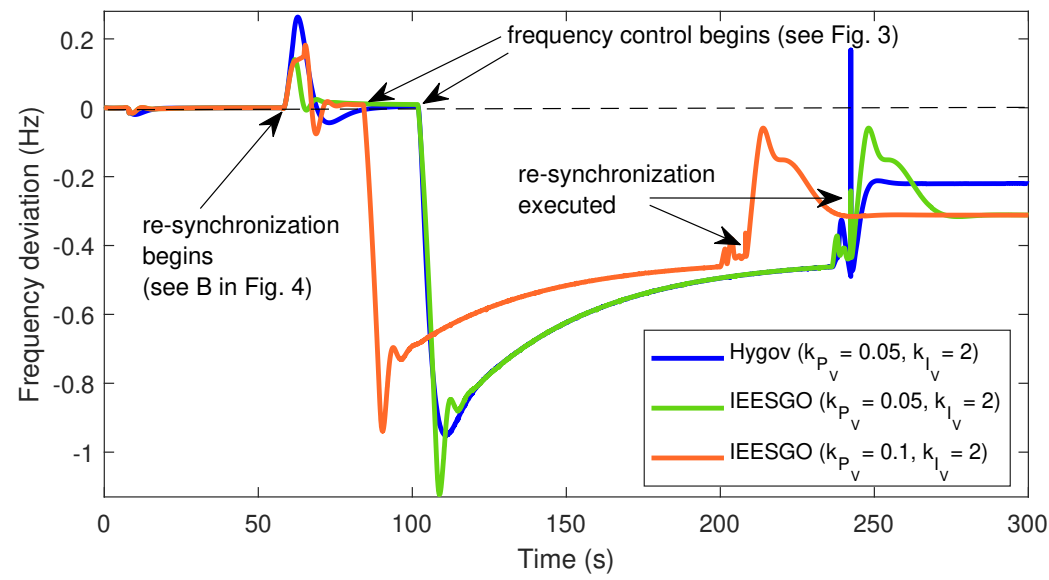

Figure 14: Distribution network frequency deviation for both IEESGO and HYGOV, for a PQ load model connected to the transmission network.

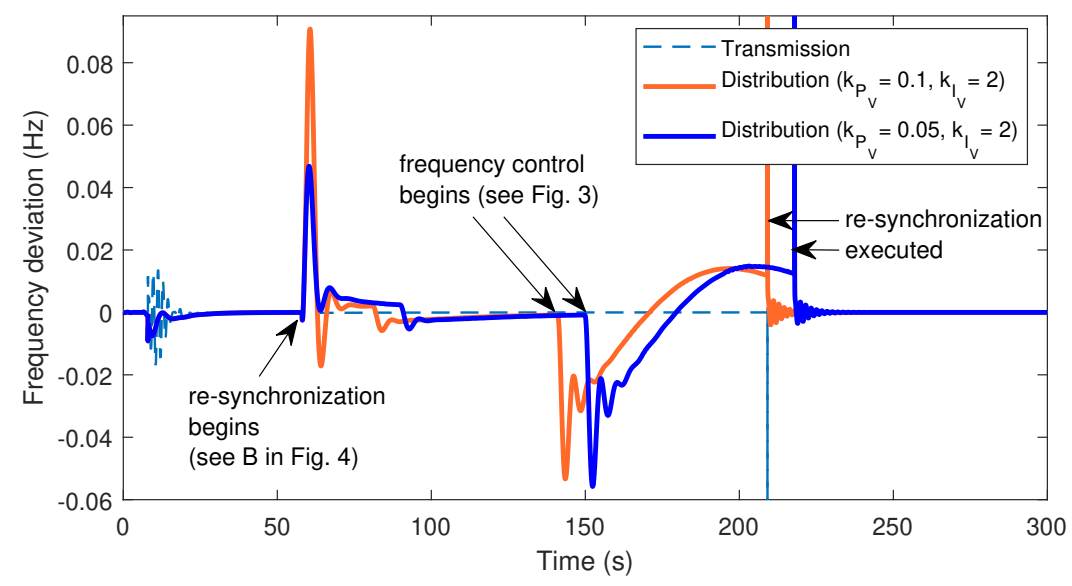

Figure 15: Frequency deviation in both transmission and distribution network for an infinite bus connected to transmission grid, with an IEESGO model in DER.

For different permanent droop of HYGOV turbine and governor system Fig. 16 plots both transmission and distribution network frequency deviations. 


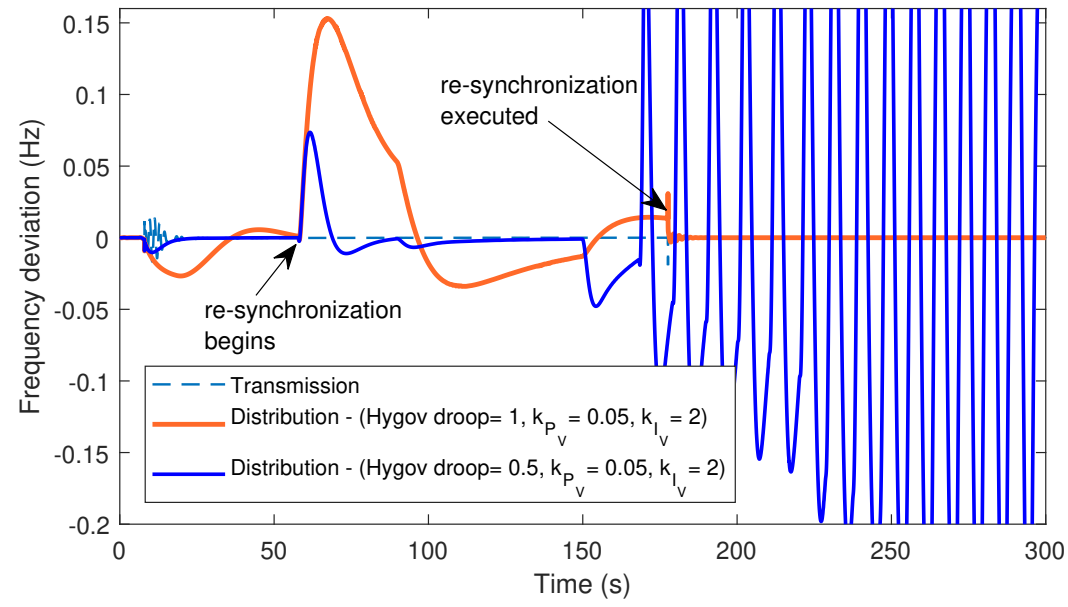

Figure 16: Frequency deviation in both transmission and distribution network for an infinite bus connected to transmission grid, with an HYGOV model in DER.

It is interesting to observe that when the droop is 1 , the control loop becomes unstable, and as a result, the controller fails to re-synchronize two grids; however, with a droop value 0.5 the controller successfully re-synchronizes two islanded grids. These results show that it is necessary to tune all control parameters, not only the ones of the new controller, but other control systems in cascade and involved in the re-synchronization process. Hence, for each kind of power plant, the parameters need to be carefully calibrated considering the turbine and governor time constants and dynamics.

\subsection{Case 3: Effect of stochastic load model on control action}

This case study is performed to analyze the performance of the proposed controller for a stochastic load model connected to the distribution network using the Modelica noise modeling [37. The stochastic load model simulates

490 the behaviour of consumer demand, showing variability when load forecasting is performed [38. This also allows considering the time-domain variability of behind-the-meter and other non-dispatchable energy sources at the distribution network level. Therefore it is necessary to investigate whether the islanded and automatic re-synchronization control actions can be executed together under aggregate load variability conditions. The stochastic load model has been simulated with standard deviation $(\sigma)$ values 0.00004 and 0.000025 ; and a period of $20 \mathrm{~ms}$.

For simulation execution we consider that the noise generation begins at $5 \mathrm{~s}$. As a result both the transmission and distribution network frequency becomes 500 stochastic as long as they are not isolated from each other. As shown in Fig 17 after the distribution network is intentionally islanded the control action begins and successfully restores the distribution network frequency deviation to zero. 
It is also interesting to observe that the transmission network frequency is deterministic in nature as long as it remains isolated from the islanded distribution grid which includes the stochastic load model. Re-synchronization occurs at $225 \mathrm{~s}$, and, again, it is observed from Fig 17 that both transmission and distribution network frequency becomes stochastic, which is as expected. Therefore this case study verifies that the proposed control architecture will successfully operate in the case of load uncertainties or non-deterministic variability of non510 dispatchable energy sources, which are becoming more common in distribution networks.

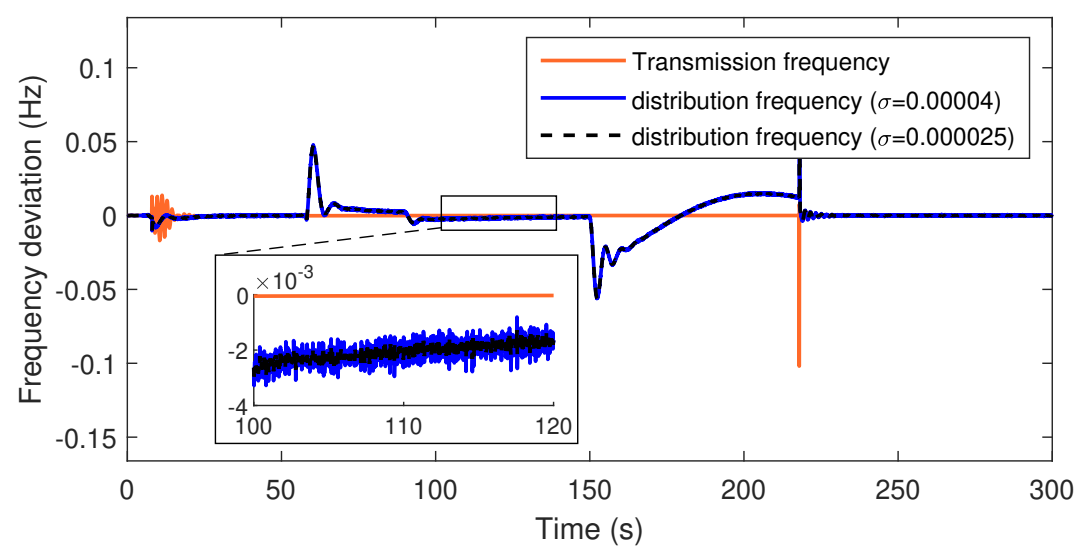

Figure 17: Transmission and distribution network frequency deviation for a stochastic load model in the distribution network.

\subsection{Case 4: Effect of delay control action}

In this section, we analyze the impact of both PMU reporting rate and data transmission delay on the performance of the proposed control scheme. Here we consider an HYGOV turbine and governor system within the DER model and, the transmission grid remains connected to the infinite bus.

To model different PMU reporting rates a ZOH from MSL (Modelica Standard Library) 39 was used. Observe from Fig 18 that delays from $50 \mathrm{~ms}$ to $150 \mathrm{~ms}$ have no major impact on the controller's performance; this is because the frequency dynamics being controlled are much larger than typical PMU reporting rates. This is a positive result, as typical PMU reporting rates are $\leq$ 16 s, i.e. 10 to 60 samples per second.

On the other hand to mimic the aggregate time-delay from a PMU device a "fixedDelay" block from MSL was used in the distribution network before this ${ }_{525}$ frequency was applied to the controller. It is interesting to observe in Fig 19 that as the data transmission delay increases (the blue trace) the control action gets delayed which eventually increases the re-synchronization time. These results are positive, as typical synchrophasor systems only incur delays in the order of 100s of milliseconds, up to a few seconds. 


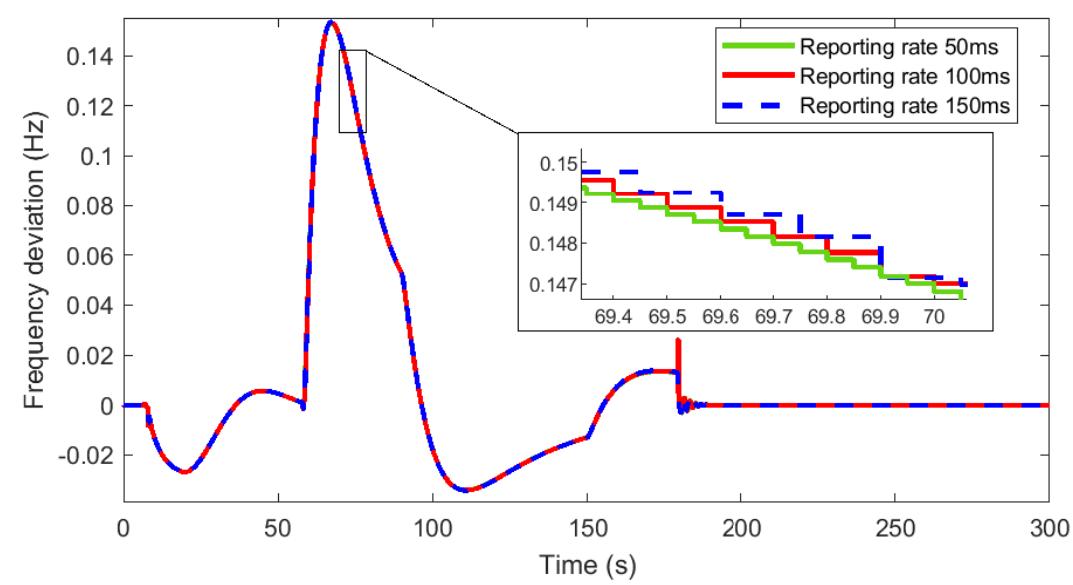

Figure 18: Distribution network frequency deviation for different PMU reporting rates.

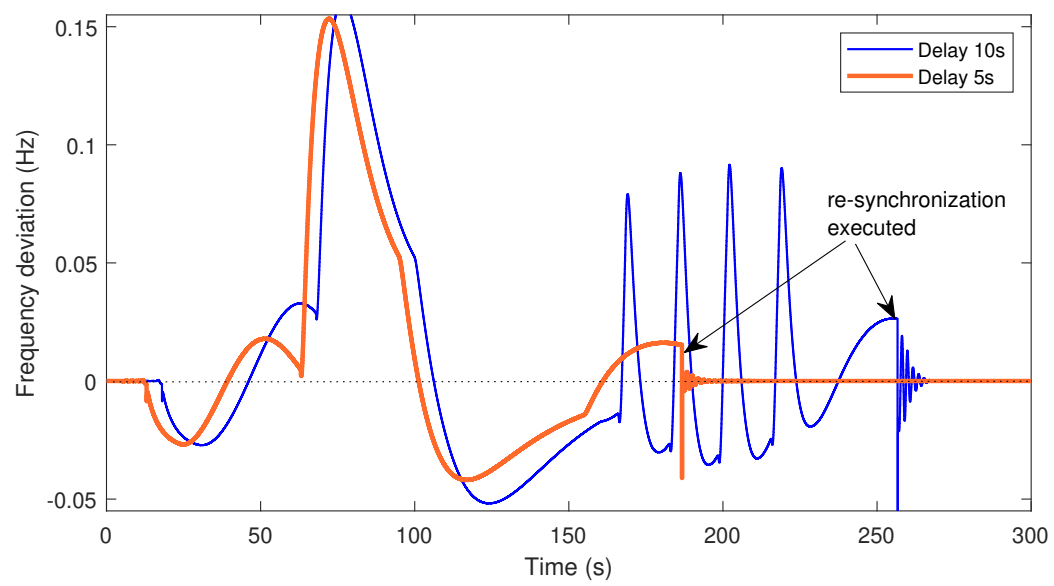

Figure 19: Distribution network frequency deviation for different delays.

530

\section{Conclusion}

An integrated and centralized control system architecture for DER operation was proposed in this work and tested in a power system model. For modeling and simulation purposes, a frequency computation approach was presented and used to emulate frequency measurements from PMUs that are utilized as input 535 to different functions of the proposed scheme.

The proposed centralized control system architecture is capable of both operating the DER in an island thereby avoiding the need of disconnecting it, and also capable of performing re-synchronization by executing the maneuver- 
ing process autonomously. Modeling the proposed control system architecture within one DG we aimed to show that, using such control architecture, it is possible to use remote measurements from synchrophasor data from different sub-stations to re-synchronize the DER autonomously. Moreover, it will only require the new control system to be installed in cascade with existing technology. In contrast, using existing technologies, it is only possible to re-synchronize 545 the system with a separate system called a re-synchronizer [8]. Similar to the author's proposal, other methods for controlled islanding [20] and synchronization [40, 21] have only been explored in simulation or lab experiments and have not been used in the field, and moreover, they also only address the control of a single generator/ DER.

${ }_{550}$ Here we have considered the PMUs located in both the transmission and the distribution network. The best locations for these PMUs in terms of their placement and delay are out of the scope of this work and will be subject to future research. By using measurements from two parts of the grid, we show how an integrated control system architecture that is interfaced in cascade with a

555 turbine's governor and excitation controls, can effectively perform two functions for which separate systems are proposed in the literature, all while at the same time without requiring the replacement the existing generator controls, but instead, complimenting them.

In this work we have only considered the grid constraints as the control/synchronizing variables which are useful for controller modeling; however, additional grid constraints (e.g. line ampacities) and cyber-physical aspects have not been considered in the modeling context.

Finally, the method can be expanded to consider multiple DERs in the distribution network, as well as distributing the control architecture to support such case. However, expanding beyond the control of a single DER is more complex. It would require an additional layer of coordination that allocates the control effort to each DER. Such work while of interest, it is outside of the scope and intent of this paper and is subject for future research.

Further work may involve tuning controllers inside the control scheme. For

570 this purpose, the controllers inside the control scheme should be re-designed for robustness to improve the overall performance.

\section{Authorship contribution statement}

Biswarup Mukherjee: Conceptualization, Methodology, Result analysis, Validation, Writing - original draft preparation and editing. Marcelo de Cas575 tro Fernandes: Validation, Writing - review \& editing. Luigi Vanfretti: Conceptualization, Methodology, Writing - review \& editing.

\section{Declaration of Competing Interest}

The authors declare that they have no known competing financial interests or personal relationships that could have appeared to influence the work reported ${ }_{580}$ in this paper. 


\section{Acknowledgements}

B. Mukherjee is supported by ARMINES, at Centre PERSEE of MINES ParisTech through the EVA project. EVA is funded in the framework of the joint programming initiative ERA-Net Smart Energy systems' focus initiative

585 Horizon 2020 research and innovation program under grant agreement No 775970.

M. Fernandes and L. Vanfretti are supported by the New York State Energy Research and Development Authority (NYSERDA) and the Engineering Research Center Program of the National Science Foundation and the Department of Energy under Award EEC-1041877, in part by the CURENT Industry Partnership Program.

\section{References}

[1] Z. Guoping, W. Weijun, M. Longbo, An overview of microgrid planning and design method, in: 2018 IEEE 3rd Advanced Information Technology, Electronic and Automation Control Conference (IAEAC), 2018, pp. 326329.

[2] X. Zhou, T. Guo, Y. Ma, An overview on microgrid technology, in: 2015 IEEE International Conference on Mechatronics and Automation (ICMA), 2015, pp. 76-81.

[7] IEEE standard for synchrophasor measurements for power systems, https : //bit.ly/314zpkt, accessed: 2021-03.

[8] M. J. Thompson, Fundamentals and advancements in generator synchronizing systems, in: 2012 65th Annual Conference for Protective Relay Engineers, 2012, pp. 203-214. doi:10.1109/CPRE.2012.6201234. 
[10] S. M. Manson, A. Upreti, M. J. Thompson, Case study: Smart automatic synchronization in islanded power systems, in: 2015 IEEE/IAS 51st Indus-

[15] C. T. F. 38.02.19, System protection schemes in power networks, technical brochure 187, Tech. rep., CIGRE International Council on large electric systems (Jun 2001).

[16] B. Yang, V. Vittal, G. T. Heydt, Slow-coherency-based controlled islanding - A demonstration of the approach on the august 14, 2003 blackout scenario, IEEE Transactions on Power Systems 21 (4) (2006) 1840-1847.

[17] A. G. Phadke, Synchronized phasor measurements in power systems, IEEE Computer Applications in Power 6 (2) (1993) 10-15.

[18] J. De La Ree, V. Centeno, J. S. Thorp, A. G. Phadke, Synchronized phasor measurement applications in power systems, IEEE Transactions on Smart Grid 1 (1) (2010) 20-27.

[19] E. Kyriakides, G. T. Heydt, Synchronized measurements in power system operation: International practices and research issues, in: 2009 IEEE Power Energy Society General Meeting, 2009, pp. 1-3. reference model, in: 2015 IEEE Eindhoven PowerTech, 2015, pp. 1-5. doi : 2015, pp. 1-10.

[11] S. D'Arco, J. A. Suul, A synchronization controller for grid reconnection of islanded virtual synchronous machines, in: 2015 IEEE 6th International Symposium on Power Electronics for Distributed Generation Sys-

12] A. B. Piardi, E. L. Geraldi, A. P. Grilo, R. Reginatto, R. A. Ramos, A control structure for smooth transfer from grid-connected to islanded operation of distributed synchronous generators, IEEE Transactions on Power Delivery 35 (2) (2019) 929-936.

microgrids using a fully distributed approach, IEEE Transactions on Smart Grid 6 (2) (2014) 943-945.

[14] N. M. Dehkordi, S. Z. Moussavi, Distributed resilient adaptive control of islanded microgrids under sensor/actuator faults, IEEE Transactions on

[20] R. Franco, C. Sena, G. N. Taranto, A. Giusto, Using synchrophasors for

9] N. A. Belyaev, Y. V. Khrushchev, S. V. Svechkarev, A. V. Prokhorov, controlled islanding - a prospective application for the uruguayan power system, IEEE Transactions on Power Systems 28 (2) (2013) 2016-2024. 
[21] T. M. L. Assis, G. N. Taranto, Automatic reconnection from intentional islanding based on remote sensing of voltage and frequency signals, IEEE Transactions on Smart Grid 3 (4) (2012) 1877-1884.

[22] A. Ortega, F. Milano, Comparison of bus frequency estimators for power system transient stability analysis, in: 2016 IEEE International Conference on Power System Technology (POWERCON), IEEE, 2016, pp. 1-6.

[23] F. Milano, A. Ortega, Frequency divider, IEEE Transactions on Power Systems 32 (2) (2016) 1493-1501.

[24] B. Mukherjee, L. Vanfretti, Modeling of PMU-based islanded operation controls for power distribution networks using Modelica and openIPSL, in: Proceedings of The American Modelica Conference, MA, USA, 2018, pp. 112-120. doi:https://doi.org/10.3384/ecp18154112.

[25] L. Vanfretti, B. Mukherjee, K. M. Moudgalya, F. J. Gómez, Automatic resynchronization controller analysis within a multi-domain gas turbine and power system model, in: 2019 7th Workshop on Modeling and Simulation of Cyber-Physical Energy Systems (MSCPES), 2019, pp. 1-5. doi : https : //doi.org/10.1109/MSCPES.2019.8738797

[26] P. Fritzson, Principles of object-oriented modeling and simulation

口 with Modelica 2.1, John Wiley \& Sons, 2010. doi:DOI:10.1109/ 9780470545669.part2.

[27] I. IEEE, IEEE/IEC international standard - measuring relays and protection equipment - part 118-1: Synchrophasor for power systems - measurements, IEC/IEEE 60255-118-1:2018 (2018) 1-78.

[28] Siemens AG. (2019) pss/e-high-performance transmission planning and analysis software, https://goo.gl/xPFpjf accessed: 2020-10.

[29] M. Tiller, Introduction to physical modeling with Modelica, Vol. 615, Springer Science \& Business Media, 2012.

[30] Modelica operators \& expressions, https://modelica.readthedocs.io/ en/latest/operators.html, accessed: 2020-10.

[31] L. Vanfretti, J. H. Chow, S. Sarawgi, B. Fardanesh, A phasor-data-based state estimator incorporating phase bias correction, IEEE Transactions on Power Systems 26 (1) (2011) 111-119. doi:10.1109/TPWRS.2010.2047031.

[32] E. Henningsson, H. Olsson, L. Vanfretti, DAE solvers for large-scale hybrid models, in: Proceedings of the 13th International Modelica Conference, Regensburg, Germany, March 4-6, 2019, Linköing University Electronic Press, 2019, pp. 491-502. doi:10.3384/ecp19157491. 
[33] L. Vanfretti, T. Rabuzin, M. Baudette, M. Murad, itesla power systems library (iPSL): A Modelica library for phasor time-domain simulations,

1) SoftwareX 5 (2016) 84 -88. doi:http://dx.doi.org/10.1016/j.softx. 2016.05 .001

[34] M. Baudette, M. Castro, T. Rabuzin, J. Lavenius, T. Bogodorova, L. Vanfretti, OpenIPSL: Open-instance power system library -update 1.5 to iTesla power systems library (iPSL): A modelica library for phasor time-domain

п simulations, SoftwareX 7 (2018) 34 - 36. doi :https://doi.org/10.1016/ j.softx.2018.01.002

[35] The Dynamic Modeling Laboratory software (Dymola), https://bit.ly/ 3iNBX3L, accessed: 2020-10.

[36] A description of DASSL: A differential/algebraic system solver, https: //www.osti.gov/servlets/purl/5882821/, accessed: 2020-10.

[37] Modelica Noise, https://github.com/DLR-SR/Noise, accessed: 2020-10.

[38] J. Zhao, Y. Bao, G. Chen, Probabilistic voltage stability assessment considering stochastic load growth direction and renewable energy generation, in: 2018 IEEE Power Energy Society General Meeting (PESGM), 2018, pp. $1-5$.

[39] Modelica Standard Library, https://github.com/modelica/ ModelicaStandardLibrary, accessed: 2021-03.

[40] M. S. Almas, L. Vanfretti, A hybrid synchrophasor and goose-based power 715 system synchronization scheme, IEEE Access 4 (2016) 4659-4668. doi: 10.1109/ACCESS . 2016.2601445. 